\title{
Patient and oncologist preferences for attributes of treatments in advanced melanoma: a discrete choice experiment
}

This article was published in the following Dove Press journal:

Patient Preference and Adherence

14 August 2017

Number of times this article has been viewed

\author{
Frank Xiaoqing Liu' \\ Edward A Witt ${ }^{2}$ \\ Scot Ebbinghaus' \\ Grace DiBonaventura \\ Beyer ${ }^{2}$ \\ Reshma Shinde' \\ Enrique Basurto ${ }^{2}$ \\ Richard W Joseph ${ }^{3}$
}

'Merck \& Co., Inc., Kenilworth, NJ, USA; ${ }^{2}$ Kantar Health, New York, NY, USA; ${ }^{3}$ Mayo Clinic, Jacksonville, FL, USA

Correspondence: Frank Xiaoqing Liu Center for Observational and Real World Evidence (CORE), Merck Research Laboratories, 35I N Sumneytown Pike, North Wales, PA 19454, USA

Tel +I 267305 I265

Email xiaoqing.liu@merck.com
Purpose: To examine and compare patient and oncologist preferences for advanced melanoma treatment attributes and to document their trade-offs for benefits with risks.

Materials and methods: A discrete choice experiment (DCE) was conducted among advanced melanoma patients and oncologists. Qualitative pilot testing was used to inform the DCE design. A series of scenarios asked stakeholders to choose between two hypothetical medications, each with seven attributes: mode of administration (MoA), dosing schedule (DS), median duration of therapy (MDT), objective response rate (ORR), progression-free survival (PFS), overall survival (OS), and grade 3-4 adverse events (AEs). Hierarchical Bayesian logistic regression models were used to determine patients' and oncologists' choice-based preferences, analysis of variance models were used to estimate the relative importance of attributes, and independent $t$-tests were used to compare relative importance estimates between stakeholders.

Results: In total, 200 patients and 226 oncologists completed the study. OS was most important to patients (33\%), followed by AEs (29\%) and ORR (25\%). For oncologists, AEs were most important (49\%), followed by OS (34\%) and ORR (12\%). An improvement from 55\% to 75\% in 1-year OS was valued similar in magnitude to a $23 \%$ decrease (from $55 \%$ to $32 \%$ ) in likelihood of AEs for oncologists.

Conclusion: Patients valued OS, AEs, and ORR sequentially as the most important attributes in making a treatment decision, whereas oncologists valued AEs most, followed by OS and ORR. In comparison, patients differed significantly from oncologists on the importance of ORR, AEs, and PFS, but were consistent in OS and the rest of attributes.

Keywords: unresectable, metastatic melanoma, systemic therapy, discrete choice experiment

\section{Introduction}

The incidence of melanoma has dramatically increased in the past three decades, with continued increases among older adults. ${ }^{1}$ Fortunately, there has been incredible progress in treatment options for patients with advanced melanoma over the past few years. These new treatments started with an anticytotoxic T-lymphocyte antigen-4, ipilimumab (Bristol-Myers Squibb, New York, NY, USA), 2,3 followed by targeted protooncogene B-Raf/mitogen-activated protein kinase (BRAF/MEK) inhibitors, including vemurafenib (Genentech Inc., San Francisco, CA, USA), ${ }^{4,5}$ dabrafenib (Novartis Pharmaceuticals Corporation, Basel, Switzerland), ${ }^{6}$ trametinib (Novartis Pharmaceuticals Corporation), ${ }^{7,8}$ and cobimetinib (Genentech Inc.), ${ }^{9}$ and then two monoclonal antibodies directed against the programmed death 1 (PD-1) receptor, pembrolizumab (Merck \& Co., Inc., Kenilworth, NJ, USA) $)^{10,11}$ and nivolumab (Bristol-Myers Squibb). ${ }^{12}$ These agents and 
their combinations ${ }^{13,14}$ have all demonstrated significant improvements in progression-free survival (PFS) and/or overall survival (OS) for patients with advanced melanoma. However, each of these new therapies carries a different benefit-to-risk profile and varies with regard to different aspects of convenience such as dosing schedule (DS) and mode of administration (MoA).

Previous research indicates that patients have the desire for decisional control and need to feel concordance between their own treatment preferences and the chosen method of treatment to improve decision-related outcomes. ${ }^{15}$ Therefore, as patients and physicians face decisions regarding treatment options for advanced melanoma, it is important to understand the preferences of both patients and physicians regarding medication attributes and to identify areas of discrepancy in order to better treat patients and increase treatment satisfaction. Furthermore, the inclusion of the patient perspective in the treatment decision-making equation is in-line with the initiatives of Institute of Medicine, National Cancer Institute, and American Society of Clinical Oncology. ${ }^{13,16,17}$

Previously, two studies have explored preferences for melanoma treatments in either patients or physicians. One study of melanoma patients in an adjuvant setting in the USA found that patients would accept high levels of toxicity for small gains in survival or avoidance of recurrence. ${ }^{18,19}$ The other study of melanoma patients and physicians in Germany again found that patients were willing to accept large side effects for even modest survival gains and to pay a steep price for therapy, whereas physicians were relatively more averse to side effects and sensitive to cost. ${ }^{19}$ However, this study focused on only one medication and had relatively low sample sizes. To our knowledge, no studies to date have yet examined how the clinical and non-clinical attributes of the most recently developed therapies for patients with advanced melanoma affect the treatment decisions for patients and oncologists.

To inform clinical practice and research community of advanced melanoma on the preferences of patients and oncologists and to potentially improve communication between patients and oncologists, this study aimed to examine and compare their preferences for advanced melanoma treatment attributes, to document their trade-offs for benefits with risks, and to explore the heterogeneity in the subgroup populations.

\section{Materials and methods}

The study aims were accomplished by deriving preference weights for patients and oncologists through a discrete choice experiment (DCE).

\section{Study sample}

Both patients and oncologists in this study completed participation through a self-administered online survey. To be eligible for the current study, all participants were required to be: 1) able to read and understand English, 2) able to operate a computer accessing the online survey, 3) located in the USA, 4) aged $\geq 18$ years, and 5) willing to provide informed consent to participate. The current study was approved by the Pearl Institutional Review Board (Indianapolis, IN, USA). Participants who were determined to be potentially eligible for the study were contacted through e-mail, with one reminder e-mail as needed.

Patient participants were recruited through the Endeavour database. Endeavour is a clinical research organization that maintains relationships with relevant advocacy groups and has previous experience working with advanced melanoma patients. To be eligible for the current study, patients were required to have a self-reported diagnosis of advanced melanoma (unresectable or metastatic) and have Eastern Cooperative Oncology Group (ECOG) performance status of $0-3 .{ }^{20}$ Patients received a US $\$ 90$ incentive for participation upon study completion.

Oncologist participants were recruited from Lightspeed Research's All Global physician panel. All Global proprietary panel members are telephone recruited to become members using hospital books and directories, medical directories, and yellow pages as original sample sources. All members undergo a double-opt in process including a registration process followed by activation through email. All Global is Safe Harbor certified and conforms to the privacy rules of the Market Research Society (MRS) and the European Society for Opinion and Market (ESOMAR) and adheres to the International Code of Marketing and Social Research Practice (ICC/ESOMAR). To be eligible for the current study, physicians were required to: 1) specialize in either medical oncology or hematology/oncology, 2) be board certified or board eligible, 3 ) have been in practice between 3 and 30 years, 4) be currently involved in providing direct patient care, 5) spend at least $50 \%$ of time in direct patient care, 6 ) provide services for an average of 100 or more cancer patients per month (including four or more advanced melanoma patients), and 7) be currently managing advanced melanoma patients with systemic treatments. Oncologists received an US\$85 incentive for participation upon study completion.

\section{Online survey}

Qualitative pilot

Draft survey instruments were first created for both patients and oncologists based on a literature review and discussion 
among the study authors. The survey instruments were then piloted in an initial qualitative study. Hour-long interviews with 12 oncologists and 8 patients were conducted through internet-mediated phone calls. The interviews assessed both comprehension of the DCE and other survey questions, as well as appropriate screening thresholds for potential patient and oncologist participants. Great care was taken during the pre-testing of the online survey instruments to ensure that the survey language was appropriate for and could be understood by respective patient and oncologist interviewees.

Initial interviews indicated that oncologists preferred to be provided with hypothetical patient information in order to make decisions during the DCE. Thus, an example patient profile was constructed by clinical experts on the study team and added to the oncologist DCE. This example profile was included in subsequent interviews with oncologists in order to receive further feedback. The profile included the following attributes: age, ECOG status, comorbidities, BRAF mutation status, lactic acid dehydrogenase (LDH) level, tumor burden, and the patient's attitude toward treatment. Physicians indicated that adding the patient profile was useful. Following the interviews, additional refinements were made to question wording, target oncologist sample size, and thresholds for respondent inclusion criteria.

\section{Discrete choice experiment}

An online DCE was used in the current study to evaluate both patients' and oncologists' stated preferences for medication characteristics. DCEs are designed to assess the relative importance or preference placed on the attributes of available medications when choosing between two medications. For example, to what degree does the efficacy of the medication affect their choice holding other attributes (eg, different MoA) constant?

The DCE estimates views on relative importance or preferences through a series of choice-based tasks. The respondent makes several hypothetical treatment decisions over a number of scenarios involving two hypothetical alternatives, which vary in terms of the levels of attributes (eg, one medication may confer $55 \%$ OS while the other may confer $75 \%$ OS). The DCE method is thought to be superior to explicitly asking for stated preferences in that it mimics a real-world situation where a medication's attributes do not occur in isolation, but in the context of other attributes. For example, one must weigh both OS and adverse events (AEs) simultaneously when considering a medication in the real world. Moreover, medications exist in the context of other available medications so oncologists and patients are also considering medication choice in the context of other available medications.
In the current study, participants completed the DCE by viewing a series of hypothetical treatment alternatives, defined by a set of seven attributes with varying levels as described above. Attributes and levels were selected based on the actual properties of available medicines, literature review, author input, and the qualitative interviews. No actual medication names were shown during the DCE. Attributes and levels (Table 1) included MoA (oral, intravenous, or subcutaneous), DS (eight levels including one or two medicines and administrations once daily, twice daily, by infusion on 2- to 3-week intervals, or by injection every 3 weeks), median duration of therapy (MDT; 3, 8, or 12 months), objective response rate (ORR; $15 \%, 35 \%$, or $65 \%$ ), PFS $(3,5$, or 11.5 months), OS $(45 \%, 55 \%$, or $75 \%)$, and grade $3-4$ toxicities/AEs $(10 \%, 32 \%$, or $55 \%)$. It is possible that some of the hypothetical treatment scenarios presented to patients and physicians contained profiles matching existing medications since, as above, part of the selection process for attributes and levels was based on the actual properties of available medicines.

Oncologists also saw a patient profile for each choice. This profile included seven attributes: patient age (under or over 65 years), ECOG status $(0-1,2$, or 3$)$, comorbid conditions (none, few, or significant), mutation status (BRAF positive or BRAF wild-type), LDH (normal or above normal), tumor burden (low or high), and patient motivation (accepts side effects or is very concerned about side effects). Steps were taken to avoid impossible or illogical attribute combinations during the process of creating patient profiles for the oncologist DCE. To achieve this, the study team received expert qualitative input from physicians to determine a set of restrictions for patient profile attributes during the pilot phase. Examples of restrictions included that a patient profile with ECOG score of 3 could not be shown with zero comorbidities, and a patient profile with significant tumor burden could not be shown with normal LDH.

The experimental design (ie, the combination of attributes and levels shown to participants) was developed using Warren Kuhfeld's D-efficient algorithm, available in Statistical Analysis System version 9.3, to ensure orthogonal design and adequate number of respondents for each combination of tasks. ${ }^{21}$ Given the number of possible combinations and the information necessary to complete each task, a full profile fractional factorial balanced incomplete block design was used. In addition, steps were taken to include a weighted adjustment for the potential impact of different patient profile attributes (eg, $<65$ or $\geq 65$ years) on oncologists' decisions about medications. This was achieved by obtaining descriptive information from physicians on attributes of 
Table I Attributes and levels in the discrete choice experiment

\begin{tabular}{|c|c|c|}
\hline Attribute & No & Levels \\
\hline \multirow[t]{3}{*}{ Mode of administration } & I & Oral - a medication taken by mouth for a period of time \\
\hline & 2 & Intravenous - an infusion given into the vein for a period of time \\
\hline & 3 & $\begin{array}{l}\text { Subcutaneous-shot given under the skin using a short needle to inject a drug into the tissue layer } \\
\text { between the skin and the muscle }\end{array}$ \\
\hline \multirow[t]{8}{*}{ Dosing schedule } & I & Two medicines; one medicine is taken twice daily, the other once daily \\
\hline & 2 & One medicine taken once daily \\
\hline & 3 & One medicine taken twice daily \\
\hline & 4 & One medicine taken by 30 -minute infusion every 3 weeks \\
\hline & 5 & One medicine taken by 60 -minute infusion every 2 weeks \\
\hline & 6 & One medicine taken by 90 -minute infusion every 3 weeks \\
\hline & 7 & $\begin{array}{l}\text { Two medicines, both are given as a } 150 \text {-minute infusion every } 3 \text { weeks for } 3 \text { months (plus/minus: } \\
\text { one of the two medicines is continued as } 60 \text {-minute infusion every } 2 \text { weeks for } 5 \text { or more months) }\end{array}$ \\
\hline & 8 & One medicine given by one injection every 3 weeks \\
\hline \multirow[t]{3}{*}{ Median duration of therapy } & I & 3 months \\
\hline & 2 & 8 months \\
\hline & 3 & 12 months \\
\hline \multirow[t]{3}{*}{ Objective response rate } & I & I5 out of 100 patients (I5\% chance of responding) \\
\hline & 2 & 33 out of 100 patients ( $33 \%$ chance of responding) \\
\hline & 3 & 65 out of 100 patients ( $65 \%$ chance of responding) \\
\hline \multirow[t]{3}{*}{ Progression-free survival } & I & 3 months \\
\hline & 2 & 5 months \\
\hline & 3 & II.5 months \\
\hline \multirow[t]{3}{*}{ Overall survival } & I & 45 out of 100 patients ( $45 \%$ of patients survive to 12 months) \\
\hline & 2 & 55 out of 100 patients ( $55 \%$ of patients survive to 12 months) \\
\hline & 3 & 75 out of 100 patients ( $75 \%$ of patients survive to 12 months) \\
\hline \multirow[t]{3}{*}{ Grade $3 / 4$ adverse events } & I & I0 out of 100 patients ( $10 \%$ likelihood of experiencing a serious side effect) \\
\hline & 2 & 32 out of 100 patients ( $32 \%$ likelihood of experiencing a serious side effect) \\
\hline & 3 & 55 out of 100 patients ( $55 \%$ likelihood of experiencing a serious side effect) \\
\hline
\end{tabular}

patients from their practices, then developing attribute distributions to identify average splits. The resulting information was integrated into the algorithm.

Ultimately, there were 99 possible DCE medication task trials that could be presented to each patient and 126 possible task trials that could be presented to each oncologist. Of these, patients responded to a total of 11 medication task trials and oncologists responded to a total of 14 trials. Figure 1 shows an example patient task trial and Figure 2 shows an example oncologist task trial.

\section{Explicit choice task}

Explicit ranking of attributes was also included in this study as an alternative assessment of preferences. Following the DCE task, participants read the written instruction "We showed various attributes or descriptions of medications in this survey. We would like you to rank order the importance of these medication attributes in making your preference decision" and asked to select the three attributes they viewed as most important. In addition, they also ranked their three chosen attributes from most (3) to least important (1).

\section{Statistical analyses}

To estimate relative preference weights for each attribute level in the DCE, Hierarchical Bayesian logistic regression models with effects coding were used. Thus, the reference level was the average across the levels in each attribute, and coefficients were estimated for each level. The resulting coefficients are viewed as part-worth utilities. Deviations of the part-worth utilities from the overall expected value were used to calculate an overall sum of squares for each attribute. Sums of squares were then divided by the attribute-specific degrees of freedom, and analysis of variance models were used to estimate the relative importance of attributes. Traditional parametric independent $t$-tests were used to assess whether the relative importance of attributes differed between patients and oncologists. Linking was used to adjust preference weights for oncologists, namely, the actual distribution of patients in an oncologist's practice was linked to the patient profiles viewed during DCE.

Finally, to assess whether preferences depend on individual characteristics for either group of stakeholders, separate models were estimated for subsets of patients and oncologists. Patients were stratified by age $(<55$ years of age or $\geq 55$ years of age), education (college degree or not), 


\begin{tabular}{|c|c|c|}
\hline Attribute & Medicine A & Medicine B \\
\hline $\begin{array}{l}\text { Mode of administration - how the } \\
\text { medicine is taken }\end{array}$ & $\begin{array}{l}\text { IV - An infusion given into the vein } \\
\text { for a period of time }\end{array}$ & $\begin{array}{l}\text { IV - An infusion given into the vein } \\
\text { for a period of time }\end{array}$ \\
\hline $\begin{array}{l}\text { Dosing schedule - frequency } \\
\text { of taking the medicine }\end{array}$ & $\begin{array}{l}\text { One medicine taken by } 30 \text {-minute } \\
\text { infusion every } 3 \text { weeks }\end{array}$ & $\begin{array}{l}\text { Two medicines, both are given as a } \\
150 \text {-minute infusion every } 3 \text { weeks } \\
\text { for } 3 \text { months (plus/minus: one of the two } \\
\text { medicines are continued as } 60 \text {-minute } \\
\text { infusion every } 2 \text { weeks for } 5 \text { or more) }\end{array}$ \\
\hline $\begin{array}{l}\text { Median duration of therapy - how long } \\
\text { the patient will be taking the medicine }\end{array}$ & 8 months & 8 months \\
\hline $\begin{array}{l}\text { Objective response rate }(\mathrm{ORR})- \\
\text { how likely the cancer will respond to } \\
\text { the medicine }\end{array}$ & 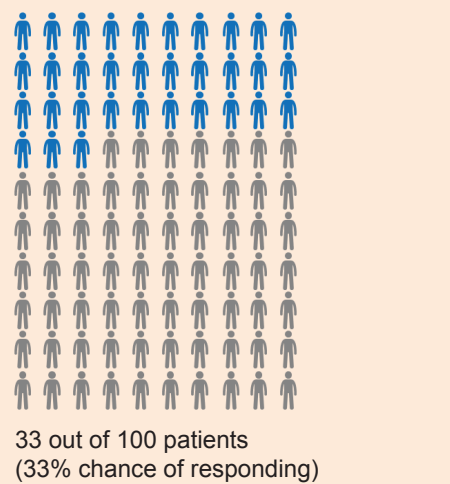 & 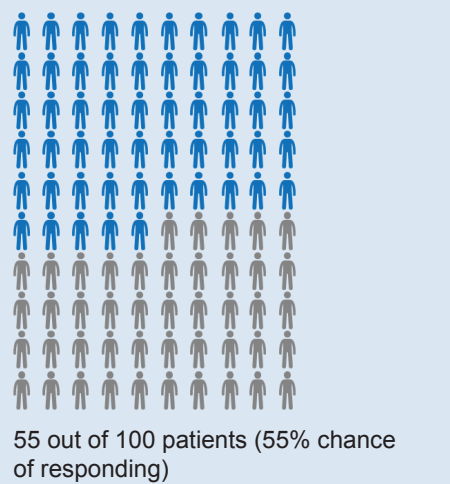 \\
\hline $\begin{array}{l}\text { Progression-free survival (PFS) - the } \\
\text { average number of months before } \\
\text { the cancer progresses although the patient } \\
\text { is being treated with the medicine }\end{array}$ & 5.5 months & 14.0 months \\
\hline $\begin{array}{l}\text { Overall survival }(\mathrm{OS}) \text { - the percentage } \\
\text { of patients taking the medicine that are } \\
\text { alive at } 12 \text { months }\end{array}$ & 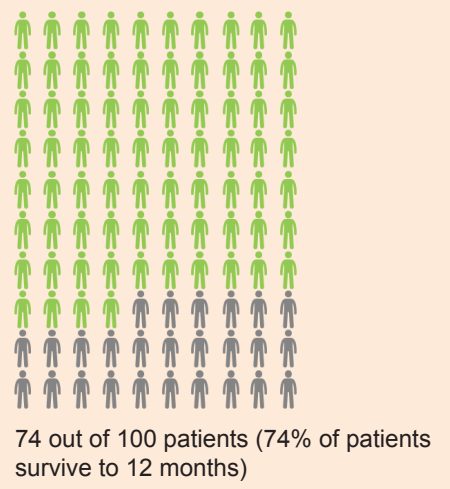 & 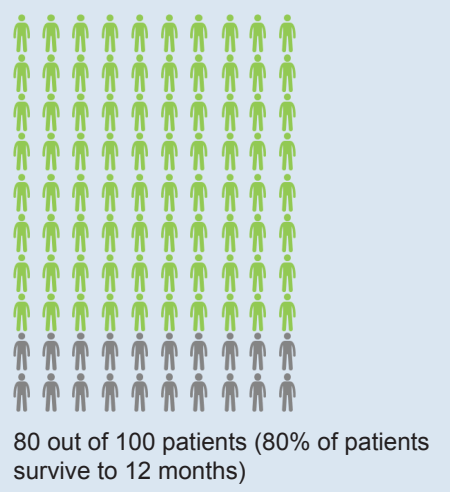 \\
\hline $\begin{array}{l}\text { Likelihood that a patient will experience } \\
\text { a side effect that requires hospitalization } \\
\text { or medical intervention while taking the } \\
\text { medicine }\end{array}$ & 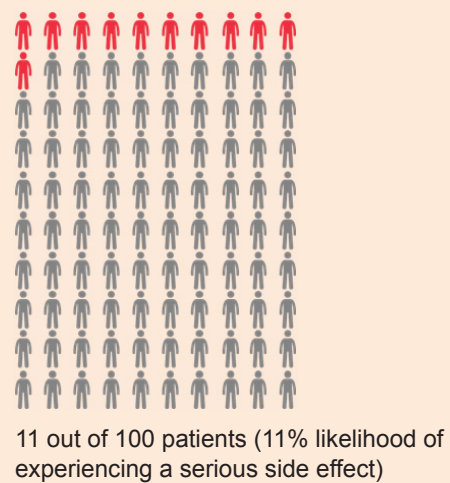 & 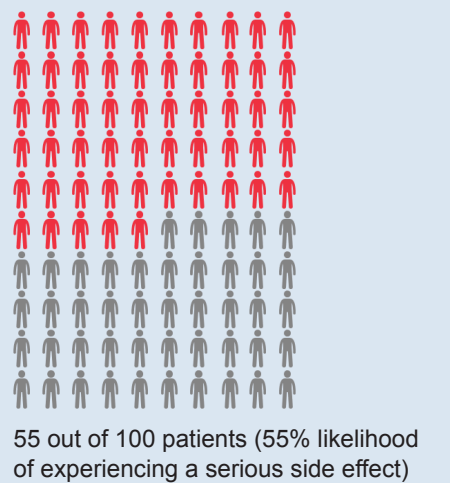 \\
\hline Select only one & Medicine A & Medicine B \\
\hline $\begin{array}{l}\text { If these were the only medicines available } \\
\text { to you, which one would you choose? }\end{array}$ & $\square$ & 口 \\
\hline
\end{tabular}

Figure I Example of patient discrete choice experiment task. Abbreviation: IV, intravenous. 


\begin{tabular}{|l|}
\hline Patient profile \\
\hline Under 65 years of age \\
\hline ECOG PS 2 \\
\hline Few manageable co-morbidities (eg, hypertension) \\
\hline BRAF wild-type \\
\hline LDH level normal \\
\hline Low tumor burden \\
\hline Patient seeks aggressive treatment, able to accept/deal with side effects \\
\hline
\end{tabular}

\begin{tabular}{|c|c|c|}
\hline Attribute & Medicine A & Medicine B \\
\hline Mode of administration & IV & IV \\
\hline Dosing schedule & $\begin{array}{l}\text { One medicine taken by } \\
30 \text {-minute infusion every } \\
3 \text { weeks }\end{array}$ & $\begin{array}{l}\text { One medicine taken by } \\
60 \text {-minute infusion every } \\
2 \text { weeks }\end{array}$ \\
\hline Median duration of therapy & 3 months & 8 months \\
\hline Objective response rate (ORR) & 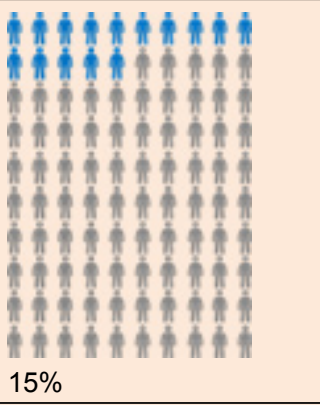 & 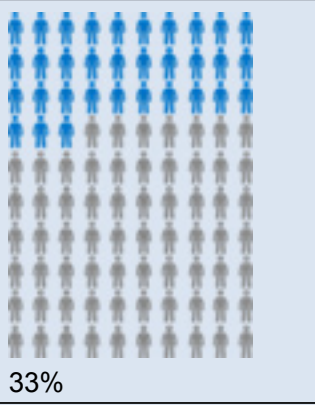 \\
\hline Progression free survival (PFS) & 3 months & 5 months \\
\hline Overall survival (OS) & 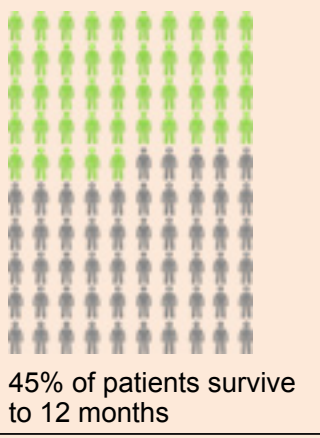 & 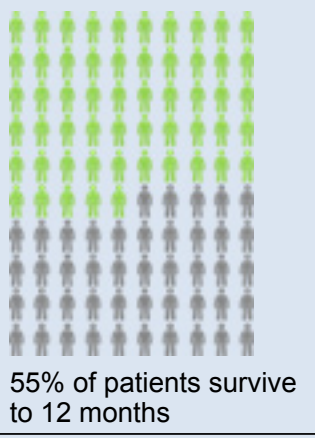 \\
\hline $\begin{array}{l}\text { Grade } 3 / 4 \text { toxicities/adverse } \\
\text { events }\end{array}$ & 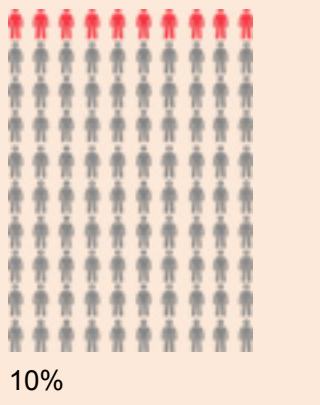 & 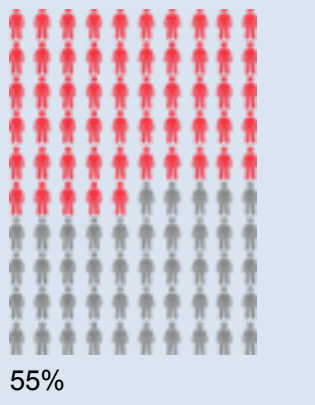 \\
\hline Select only one & Medicine A & Medicine B \\
\hline $\begin{array}{l}\text { If these were the only } \\
\text { medications available to you for } \\
\text { first line prescribing for this } \\
\text { patients with advanced } \\
\text { (unresectable/metastatic) } \\
\text { melanoma, which one would } \\
\text { you choose? }\end{array}$ & $\square$ & $\square$ \\
\hline
\end{tabular}

Figure 2 Example of oncologist discrete choice experiment task.

Abbreviations: BRAF, B-Raf; ECOG PS, Eastern Cooperative Oncology Group Performance Status; IV, intravenous; LDH, lactic acid dehydrogenase. 
income (household income above or below US\$75,000), and location (urban or rural). Oncologists were stratified by work context (self-identified setting as academic or non-academic), years in practice ( $<15$ years or $\geq 15$ years), monthly metastatic melanoma patient load $(<21$ patients or $\geq 21$ ), and location (urban or rural). Sample medians were used to determine cut-off points for income, years in practice, and metastatic melanoma patient load. Independent sample $t$-tests were used to compare strata in each subset analysis.

\section{Results}

Responses were monitored during data collection, and participants with high levels of choice instability or inconsistency in the DCE were replaced in the data set (accounting for $1.2 \%$ of potentially eligible patients and $3.6 \%$ of potentially eligible oncologists replaced). The targeted enrollment for this study was 200 patients and 225 oncologists and recruitment was halted once those numbers were achieved. In total, 200 patients and 226 oncologists $(73.2 \%$ and $62.4 \%$ of respondents, respectively) met all inclusion criteria (including demonstrating choice stability and consistency in the DCE), and completed the study.

\section{Participant characteristics}

A total of 935 patients in the Endeavour database were potentially eligible for the study and approached, of whom $273(29.2 \%$ of 935$)$ completed the electronic informed consent form for study participation and underwent further screening. About 224 (82.1\% of 273) patients were eligible for the study and 49 (17.9\% of 273) were not; 211 (94.2\% of 224) patients completed the online survey and $13(5.8 \%$ of 224) withdrew while taking the online survey. As above, 11 patients demonstrated choice instability or inconsistency during DCE tasks and their data were replaced by those of a subsequent participant.

The mean age of the patient sample was 46.52 years. The majority of patient participants were female $(60.5 \%)$, married (68.5\%), White (83.5\%), had at least a college degree (73.0\%), employed (62.5\%), and had a household income of US $\$ 75,000$ or higher (55.0\%). Almost half had employer provided insurance (46.0\%), and only $2.0 \%$ had no insurance. Of those who had insurance, the majority reported that it covered everything but copay (54.0\%).

A total of 1,113 physicians in the All Global physician panel were identified as potentially eligible for the study and approached; $362(32.5 \%$ of 1,113$)$ physicians completed the electronic informed consent form for study participation and underwent further screening. About 271 (74.9\% of 362) physicians were eligible for the study and 91 (25.1\% of 362$)$ were not; 239 (88.2\% of 271$)$ completed the survey and 32 (11.8\% of 271) withdrew while taking the survey. As above, 13 oncologists demonstrated choice instability or inconsistency during DCE tasks and their data were replaced.

The majority of oncologists were male (82.3\%) and aged $\leq 50(63.3 \%)$ years. An approximately equal number of oncologists specialized in medical oncology (50.4\%) and hematology oncology (49.6\%), and had been practicing for 15.4 years on average. The majority practiced in office-based private groups (62.8\%). Oncologists reported that the majority of their time was spent in direct patient care (93.2\%) and saw an average of 342.6 cancer patients in a typical month, of whom an average of 120.0 had "advanced melanoma."

\section{Attribute level preferences}

Full DCE regression results for both patients and oncologists are represented graphically in Figures 3 and 4.

In the DCE, patients indicated the strongest preferences among the levels of OS, followed by AEs, ORR, and PFS (Figure 4). In contrast, preferences among levels were relatively undifferentiated for MoA, DS, and MDT. Oncologists indicated the strongest preferences among the levels of AEs, followed by OS, ORR, and PFS. They also showed relatively undifferentiated preferences among the levels of MoA, DS, and MDT (Figure 3). Patients and oncologists significantly differed in the strength of their preferences. Of particular interest, patient preferences indicated more differentiation for OS, ORR, and PFS.

Subgroup analyses indicated small, but significant differences in patient and oncologist preferences across attribute levels. Namely, patients with incomes of $<$ US\$75,000 had more differentiated preferences for OS, PFS, and ORR as compared to patients with incomes of $\geq \mathrm{US} \$ 75,000$. Patients living in rural areas also showed more differentiated preferences for PFS and ORR compared with those living in urban areas. Patient age and education were not associated with differential attribute level preferences. Oncologists working in academic settings had more differentiated preferences for OS, DS, and MoA as compared to those in non-academic settings, whereas oncologists seeing fewer advanced melanoma patients had more differentiated preferences for AEs, and those in urban areas were more differentiated on DS compared with those in rural areas. Years in practice was not associated with differential attribute level preferences. 


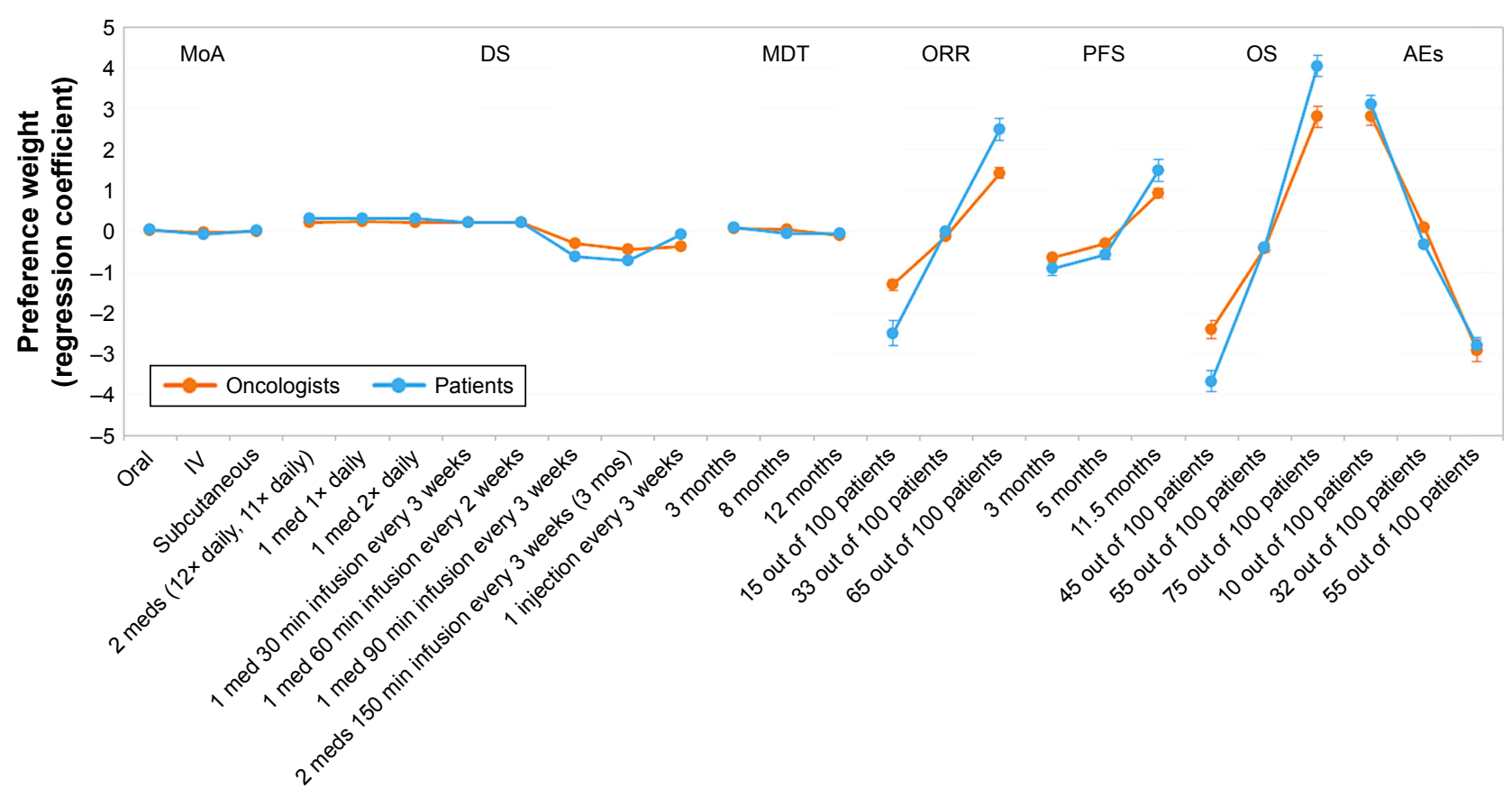

Figure 3 Relative preference weights for patients and oncologists, as estimated by Hierarchical Bayesian logistic regression models with effects coding. Abbreviations: AEs, adverse events; DS, dosing schedule; IV, intravenous; MDT, median duration of therapy; MoA, mode of administration; med, medication; OS, overall survival; ORR, objective response rate; PFS, progression-free survival.

\section{Relative attribute preferences}

On the basis of the DCE, patients viewed OS as most important (Figure 4). AEs and ORR were also viewed as relatively important by patients, whereas PFS and DS were viewed as moderately important, and MDT and MoA were viewed as of lower importance. Oncologists viewed AEs as most important, followed by OS (Figure 4). ORR, PFS, and DS were viewed as moderately important, and MDT and MoA were viewed as of lower importance. Comparatively speaking, oncologists viewed AEs $(P<0.001)$ as of significantly higher relative importance than patients, whereas patients viewed both ORR $(P<0.001)$ and PFS $(P<0.001)$ as of significantly higher relative importance than oncologists.

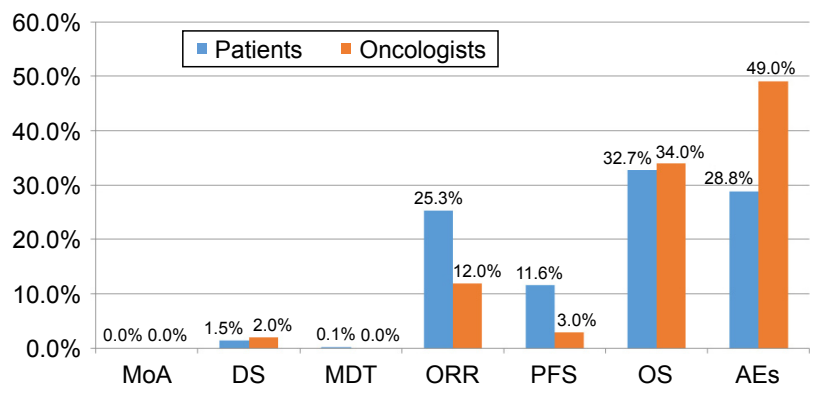

Figure 4 Relative importance of attributes for patients and oncologists. Abbreviations: AEs, adverse events; DS, dosing schedule; MDT, median duration of therapy; MoA, mode of administration; ORR, objective response rate; OS, overall survival; PFS, progression-free survival.

\section{Explicit preferences}

When asked directly to state their preferences, patients were most likely to select OS (61.5\%), ORR (49.5\%), and PFS $(43.0 \%)$ as important and OS ( mean $=2.3)$, ORR $($ mean $=2.2)$, and MDT (mean $=2.0$ ) were ranked as most important among patients who selected those attributes. Oncologists were most likely to select OS (85.0\%), PFS (74.3\%), and AEs $(64.6 \%)$ as most important. OS (mean $=2.7$ ) was ranked as most important among oncologists who selected it as one of the three most important attributes.

\section{Discussion}

The study suggests that patients place high value on therapeutic outcomes. Patient preferences indicated that optimizing OS was most important, followed by minimizing grade 3-4 toxicities/AEs and maximizing ORR. In contrast, oncologists weighed incidence of grade 3-4 toxicities/AEs as relatively most important, followed by OS and ORR. This preference for OS among patients and for minimizing toxicities/AEs among oncologists is consistent with existing research in melanoma and cancer more generally. ${ }^{18,19,22-24}$ Consistent with research indicating that clinical characteristics of oncologists are associated with preferences, ${ }^{25}$ academic oncologists showed more differentiated preferences among levels of OS, DS, and MoA, whereas oncologists treating fewer advanced melanoma patients were more 
differentiated for incidence of grade 3-4 toxicities/AEs, and urban oncologists were more differentiated on DS.

Patients' explicitly rated preferences were somewhat consistent with the DCE. However, although incidence of grade 3-4 toxicities/AEs events was second most important in the DCE, it was only selected as one of the three most important attributes by $32.5 \%$ of patients (ranked 4 th). This discrepancy suggests that patients may not be fully aware of the utility they place on avoidance of grade 3-4 toxicities/ AEs and presents a challenge for patients in terms of identifying their most preferred treatment and communicating about treatment options with health care professionals.

The preferences reported by oncologists when asked explicitly differed from the choices they made in the DCE. Namely, when explicitly stating importance, oncologists were more likely to select OS and PFS than incidence of grade 3-4 toxicities/AEs, which was the most important attribute based on the DCE. This discrepancy between explicitly held preferences and choice-based preferences has implications for medical decision making and patient-physician communication. Oncologists may believe, and therefore communicate to patients, that they select medication and treatment plans weighing survival factors most heavily, when in fact incidence of toxicities/AEs may be more important.

Patients and oncologists differed in their preferences for advanced melanoma treatment attributes based both on DCE and explicit ratings. In particular, in the preference elicitation task, patients indicated more differentiation, signaling stronger preferences, for OS, ORR, and PFS as compared to oncologists. In addition, comparison of the relative importance of attributes based on DCE task indicated that patients weighed OS as most important, whereas oncologists favored incidence of grade 3-4 toxicities/AEs. Indeed, oncologist ascribed significantly more relative importance to AEs compared to patients, whereas patients ascribed significantly more relative importance to ORR and PFS. Notably, patients and oncologists were both most likely to select OS as important when asserting explicit preferences. This pattern of results suggests that oncologists' selections of treatment for advanced melanoma may not align with patients' preferences, and furthermore that discussions between patients and oncologists may be unlikely to uncover these differences as both explicitly favor OS.

To our knowledge, this is the first study to assess both discrete choice and explicit treatment preferences of advanced melanoma patients and oncologists. The insights gained through this evidence-based study about the acceptable tradeoffs within patients and oncologists, comparisons between patients and oncologists, and the discrepancies between explicit and implicit preferences of these stakeholders may help support the ongoing development of patient-centered care among cancer patients. Specifically, insights may better patients' and oncologists' communication to help make their treatment-related decisions align with patients' needs and desires. Several studies of preferences for the treatment of non-melanoma cancer are consistent with the results presented here, in that they also indicate that patients may be more willing to accept side effects and are less sensitive to cost than physicians ${ }^{22-24,26}$ and that individual characteristics of the patient or physician can influence preferences. ${ }^{25-27}$ Awareness of these inconsistencies can improve shared medical decision making, and thus, improve patient satisfaction with treatment.

\section{Limitations}

This study had several limitations. The preferences expressed by the patients and oncologists in this study may not generalize to all members of these stakeholder groups as participants were subject to selection criteria based on individual and clinical characteristics. Results may also not generalize to those without Internet access and/or patients with high symptom burden. In addition, choices made in the preference elicitation task were in response to hypothetical choice profiles, and therefore do not carry the same clinical, financial, or emotional consequences as actual decisions. ${ }^{25}$ Furthermore, although the attributes and levels were selected based on review of the literature and current treatment landscape, it is possible that some clinical attributes that could influence choices were not included in the design. However, it would have been detrimental to experimental design to specify all possible distinct toxicities/AEs. Thus, although the literature shows that some toxicities/AEs may be valued differently compared to others by patients and/or physicians, ${ }^{28}$ we were unable to determine the precise influence of every possible toxicity/AE on study results. Rather, we were only able to assess holistic, hypothetical effects. In relation, this study did not include cost-related concerns and instead only focused on risks, benefits, and convenience. In addition, although the subgroup analyses examined possible individual difference characteristics that could affect preferences, it is possible that other patient or oncologist characteristics not included here could be associated with preferences. Finally, the response rate for patients was slightly lower than that of other studies that surveyed melanoma survivors without advanced disease by mail. ${ }^{29}$ However, it was similar to response rates reported for cancer patients with more advanced disease, 
which confers morbidities that may make it more difficult to respond. ${ }^{30}$ For oncologists, the response rate was consistent with that of other recent studies in which oncologists completed online surveys. ${ }^{31}$

Ultimately, understanding similarities and differences in patients' and oncologists' viewpoints on aspects of treatment for advanced melanoma may help oncologists make value-based decisions. It is important to understand the preferences of both patients and oncologists regarding advanced melanoma treatment options to better treat patients and increase treatment satisfaction and adherence. Findings from this DCE study can be used as a basis for health care providers to discuss treatment tradeoffs with patients as a way to increase shared decision making. Future research should seek to qualify how similarities and differences in patients' and health care providers' viewpoints affect actual treatment selection and associated patient outcomes.

\section{Acknowledgments}

The authors thank Michelle Vichnin, Tarek Hammad, and Cathy Anne Pinto, employees of Merck \& Co., Inc., who assisted in the implementation of the study. Salome Samant, an employee of Merck \& Co., Inc., assisted with the preparation of the manuscript. EAW is now at Walgreens. This research was financially supported by Merck \& Co., Inc. Kenilworth, NJ, USA.

This paper was presented at Society for Melanoma Research (SMR) 2016 Congress, November 6-9, 2016, Boston, MA, USA as poster presentations with independent results for patients and oncologists and at National Comprehensive Cancer Network (NCCN) 22nd Annual Conference, March 23-25, 2017, Orlando, FL, USA as a poster presentation with comparative results between the two stakeholders.

\section{Author contributions}

FXL, SE, GDBB, EB, and RWJ involved in the conception and design of the study. All authors contributed to the analysis and interpretation of the data, in critical review and revision of manuscript, and for the final approval of manuscript.

\section{Disclosure}

FXL, SE, and RS are employees of Merck \& Co., Inc., Kenilworth, NJ, USA, a manufacturer that funded this study and produces medication therapy used to treat melanoma. GDBB, EB, and EAW are employees of Kantar Health. Kantar Health received funding from Merck and Co. Inc., Kenilworth, NJ, USA, for conducting this study and the development of this manuscript. RWJ served as a paid consultant for the study project for Merck \& Co., Inc., Kenilworth, NJ, USA, but received no compensation for the development of this manuscript. The authors report no other conflicts of interest in this work.

\section{References}

1. Cancer Facts \& Figures 2016. Atlanta, GA: American Cancer Society; 2016. Available from: https://www.cancer.org/content/dam/cancerorg/research/cancer-facts-and-statistics/annual-cancer-facts-andfigures/2016/cancer-facts-and-figures-2016.pdf. Accessed October 28, 2016.

2. Hodi FS, O'Day SJ, McDermott DF, et al. Improved survival with ipilimumab in patients with metastatic melanoma. NEngl J Med. 2010; 363(8):711-723.

3. Robert C, Thomas L, Bondarenko I, et al. Ipilimumab plus dacarbazine for previously untreated metastatic melanoma. $N$ Engl J Med. 2011; 364(26):2517-2526.

4. Chapman PB, Hauschild A, Robert C, et al; BRIM-3 Study Group. Improved survival with vemurafenib in melanoma with BRAF V600E mutation. N Engl J Med. 2011;364(26):2507-2516.

5. McArthur GA, Chapman PB, Robert C, et al. Safety and efficacy of vemurafenib in BRAF(V600E) and BRAF(V600K) mutation-positive melanoma (BRIM-3): extended follow-up of a phase 3, randomised, open-label study. Lancet Oncol. 2014;15(3):323-332.

6. Hauschild A, Grob JJ, Demidov LV, et al. Dabrafenib in BRAF-mutated metastatic melanoma: a multicentre, open-label, phase 3 randomised controlled trial. Lancet. 2012;380(9839):358-365.

7. Robert C, Karaszewska B, Schachter J, et al. Improved overall survival in melanoma with combined dabrafenib and trametinib. N Engl J Med. 2014;372(1):30-39.

8. Long GV, Stroyakovskiy D, Gogas H, et al. Dabrafenib and trametinib versus dabrafenib and placebo for Val600 BRAF-mutant melanoma: a multicentre, double-blind, phase 3 randomised controlled trial. Lancet. 2015;386(9992):444-451

9. Larkin J, Ascierto PA, Dréno B, et al. Combined vemurafenib and cobimetinib in BRAF-mutated melanoma. NEngl J Med. 2014;371(20): 1867-1876.

10. Robert C, Schachter J, Long GV, et al; KEYNOTE-006 investigators. Pembrolizumab versus Ipilimumab in advanced melanoma. $N$ Engl $J$ Med. 2015;372(26):2521-2532.

11. Ribas A, Puzanov I, Dummer R, et al. Pembrolizumab versus investigator-choice chemotherapy for ipilimumab-refractory melanoma (KEYNOTE-002): a randomised, controlled, phase 2 trial. Lancet Oncol. 2015;16(8):908-918.

12. Robert C, Long GV, Brady B, et al. Nivolumab in previously untreated melanoma without BRAF mutation. $N$ Engl J Med. 2015;372(4): 320-330.

13. Larkin J, Chiarion-Sileni V, Gonzalez R, et al. Combined nivolumab and ipilimumab or monotherapy in untreated melanoma. $N$ Engl J Med. 2015;373(1):23-34.

14. Postow MA, Chesney J, Pavlick AC, et al. Nivolumab and ipilimumab versus ipilimumab in untreated melanoma. NEngl J Med. 2015;372(21): 2006-2017.

15. Brown R, Butow P, Wilson-Genderson M, Bernhard J, Ribi K, Juraskova I. Meeting the decision-making preferences of patients with breast cancer in oncology consultations: impact on decision-related outcomes. J Clin Oncol. 2012;30(8):857-862.

16. Wolchok JD, Kluger H, Callahan MK, et al. Nivolumab plus ipilimumab in advanced melanoma. N Engl J Med. 2013;369(2):122-133.

17. Kelly C. ASCO cancer drug valuation tool will incorporate patient preferences. Pinksheet. Available from: https://pink.pharmamedtechbi. com/PS079469/ASCO-Cancer-Drug-Valuation-Tool-Will-IncorporatePatient-Preferences. Published 2016. Accessed April 11, 2016.

18. Kilbridge KL, Weeks JC, Sober AJ, et al. Patient preferences for adjuvant interferon alfa-2b treatment. J Clin Oncol. 2001;19(3):812-823. 
19. Krammer R, Heinzerling L. Therapy preferences in melanoma treatment - willingness to pay and preference of quality versus length of life of patients, physicians and healthy controls. PLoS One. 2014; 9(11):e111237.

20. Oken M, Creech R, Tormey D, et al. Toxicity and response criteria of the Eastern Cooperative Oncology Group. Am J Clin Oncol. 1982;5(6): 649-656.

21. Kuhfeld W. Marketing Research Methods in SAS: Experimental Design, Choice, Conjoint, and Graphical Techniques. Available from: https://support.sas.com/techsup/technote/mr2010.pdf. Published 2010. Accessed April 20, 2017.

22. Shafey M, Lupichuk SM, Do T, Owen C, Stewart DA. Preferences of patients and physicians concerning treatment options for relapsed follicular lymphoma: a discrete choice experiment. Bone Marrow Transplant. 2011;46(7):962-969.

23. Kunneman M, Pieterse AH, Stiggelbout AM, et al. Treatment preferences and involvement in treatment decision making of patients with endometrial cancer and clinicians. Br J Cancer. 2014;111(4):674-679.

24. Arellano J, Hauber AB, Mohamed AF, et al. Physicians' preferences for bone metastases drug therapy in the United States. Value Health. 2015; 18(1):78-83.

25. Nathan H, Bridges JF, Schulick RD, et al. Understanding surgical decision making in early hepatocellular carcinoma. J Clin Oncol. 2011; 29(6):619-625.
26. daCosta DiBonaventura M, Copher R, Basurto E, Faria C, Lorenzo R. Patient preferences and treatment adherence among women diagnosed with metastatic breast cancer. Am Heal Drug Benefits. 2014; 7(7):386-396.

27. Wong YN, Egleston BL, Sachdeva K, et al. Cancer patients' trade-offs among efficacy, toxicity, and out-of-pocket cost in the curative and noncurative setting. Med Care. 2013;51(9):838-845.

28. Korfage IJ, de Koning HJ, Habbema JDF, Schröder FH, Essink-Bot ML. Side-effects of treatment for localized prostate cancer: are they valued differently by patients and healthy controls? BJU Int. 2007;99(4):801-806.

29. Glenn BA, Chen KL, Chang LC, Lin T, Bastani R. Skin examination practices among melanoma survivors and their children. J Cancer Educ. 2017;32(2):335-343.

30. Smith T, Stein KD, Mehta CC, et al. The rationale, design, and implementation of the American Cancer Society's studies of cancer survivors. Cancer. 2007;109(1):1-12.

31. Muscaritoli M, Rossi Fanelli F, Molfino A. Perspectives of health care professionals on cancer cachexia: results from three global surveys. Ann Oncol. 2016;27(12):2230-2236.
Patient Preference and Adherence

\section{Publish your work in this journal}

Patient Preference and Adherence is an international, peer-reviewed, open access journal that focuses on the growing importance of patient preference and adherence throughout the therapeutic continuum. Patient satisfaction, acceptability, quality of life, compliance, persistence and their role in developing new therapeutic modalities and compounds to optimize

\section{Dovepress}

clinical outcomes for existing disease states are major areas of interest for the journal. This journal has been accepted for indexing on PubMed Central. The manuscript management system is completely online and includes a very quick and fair peer-review system, which is all easy to use. Visit http://www. dovepress.com/testimonials.php to read real quotes from published authors. 